\title{
Sitting position in neurosurgery: does venous air embolism prolong length of stay in neurosurgical intensive care unit?
}

\author{
Matas M., Sekulić A., Zlatar P. ; University Hospital Centre Zagreb, Department of \\ Anaesthesiology and Intensive Care, Zagreb, Croatia
}

Box \& Whisker Plot: ICU days vs. air embolism

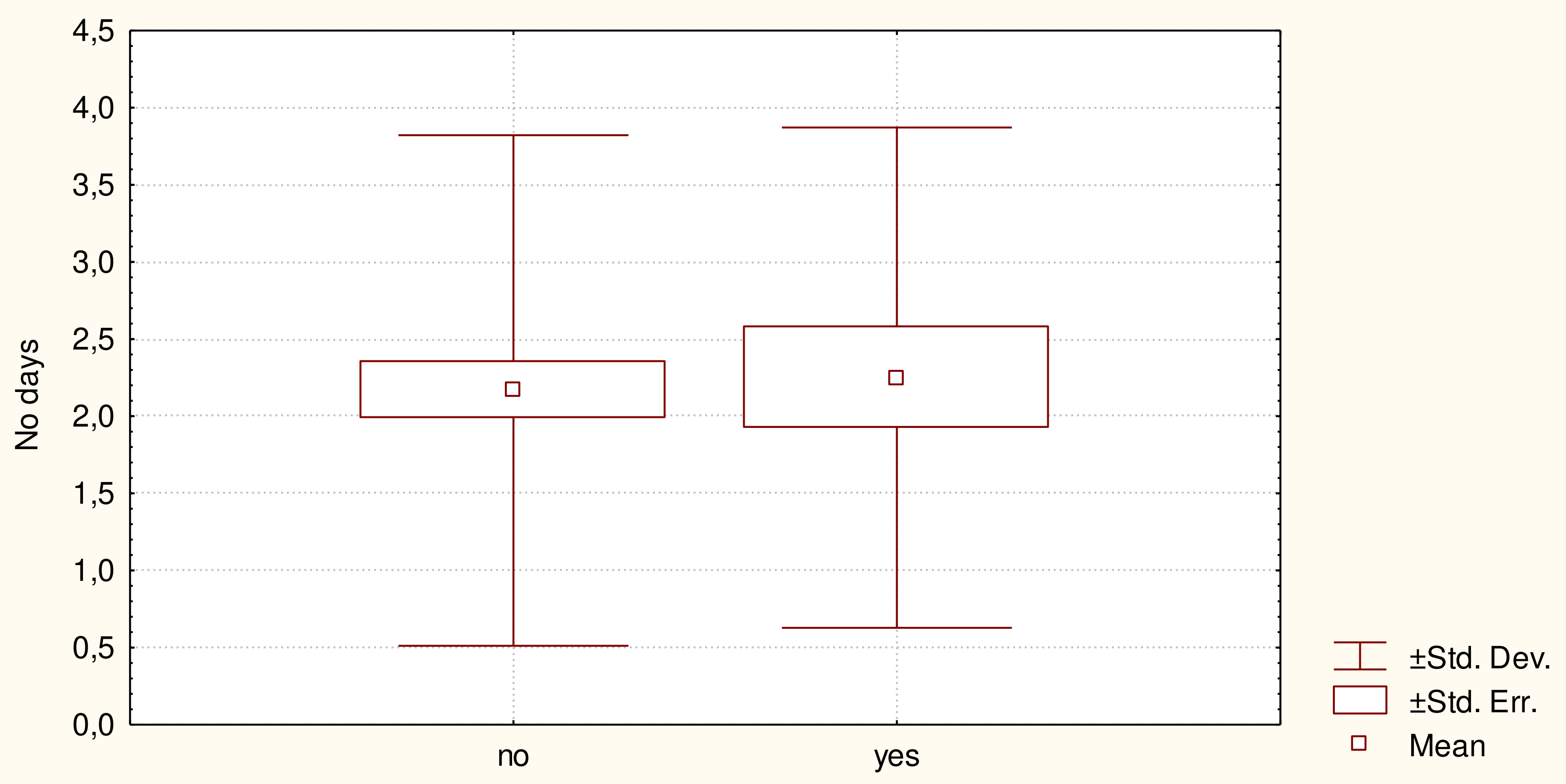

Background and Goal of Study: Sitting position in neurosurgery is used in posterior cranial fossa and medulla oblongata surgery. One of the most common and most severe complication of operations in this position is venous air embolism (VAE). Incidence varies depending on the method used to detect VAE, being as high as up to $76 \%$ when transesophageal echocardiography monitoring is used. (1)

The aim of this study was to determine the difference in duration of stay in neurosurgical intensive care unit (NICU) for patients operated in sitting position with and without venous air embolism.

Materials and methods: In our study we included 114 patients of all ages operated in sitting position from January 2011 to May 2015. All patients underwent total intravenous anesthesia with thiopental/propofol and fentanyl, and they all had central venous catheter. VAE monitoring included clinical acknowledgement (sudden decrease in end-tidal $\mathrm{CO} 2$, sudden decrease in mean arterial pressure, increase in difference between end-tidal and arterial $\mathrm{CO} 2$ ).
Results and discussion: VAE was observed in 26 out of 114 patients in sitting position. The duration of their stay in NICU was in range of 1 to 13 days $(2,65 \pm 2,62)$ versus patients without VAE, range 1 to 29 days $(2,60 \pm 3,47)$. To test our hypothesis we used t-test $(t=0,0679$, $\mathrm{P}=0,9460)$.

Conclusion: We have found no statistical difference in the length of stay in the NICU between patients who have suffered from VAE during sitting position neurosurgery and those who have not.

Reference: (1) Porter JM, Pidgeon C, Cunningham AJ Br J Anaesth. 1999; 82(1):117-28 